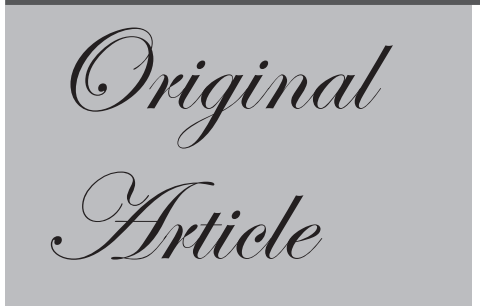

${ }^{1}$ Department of Gastroenterology, Topiwala National Medical College and BYL Ch Hospital, Dr A.L Nair Road, Mumbai-400 008, Maharashtra, India.

${ }^{2}$ Department of Pathology, Lokmanya Tilak Municipal Medical College and General Hospital, Mumbai, Maharashtra, India

Corresponding Author:

Dr Nikhil Sonthalia

Email:nikhil_zenith@yahoo.co.in

\section{Significance of Z-Line Appearance Grading in Patients of Gastroesophageal Reflux Disease}

\author{
Nikhil Sonthalia ${ }^{1}$, Samit S Jain ${ }^{1}$, Qais Contractor ${ }^{1}$, \\ Vinay Zanwar ${ }^{1}$, Vinay B Pawar ${ }^{1}$, Ravindra G Surude ${ }^{1}$, \\ Anjali Amarapurkar ${ }^{2}$, Pravin M Rathi ${ }^{1}$
}

ABSTRACT

Background and Aim: Z-line appearance grading (ZAP) is a validated system that correlates with prevalence of intestinal metaplasia (IM) in gastroesophageal reflux disease (GERD). The aim was to determine the significance of ZAP grading in GERD patients.

Materials and Methods: In this prospective cross-sectional hospital based case-control study, 109 consecutive GERD patients diagnosed by GERD questionnaire and 102 controls were included. On endoscopy GERD was classified as erosive reflux disease (ERD) $(n=42)$ and non-erosive reflux disease (NERD) $(n=67)$. Los Angeles (LA) grading and ZAP grading were done. Biopsies were taken from lower esophagus, Z-line, gastric cardia, gastric corpus and antrum incases. Findings were correlated with ZAP grading.

Results: Controls had significantly more ZAP grade '0' (79.41\% vs $21.11 \%$ ) and less ZAP grade I, II and III (20.58\% vs $78.89 \%)$ as compared to cases $(\mathrm{P}=0.0001)$. Microscopic esophagitis was seen in all ZAP III GERD patients and was significantly more in ZAP II as compared to ZAP I $(97.67 \%$ vs $71.055, \mathrm{p}=0.0023)$. ZAP grading correlated significantly with LA grading (Spearman correlation factor $=0.478, \mathrm{p}<0.01$ ). The likelihood ratio of ZAP grade to diagnose NERD was 3.26 in cases. At the lower esophagus, IM was significantly more with higher grades (III vs II) of ZAP.

Conclusion: ZAP grading is useful in diagnosing GERD, especially NERD in subjects with positive GERD symptom score.Microscopic esophagitis and IM is more with a higher ZAP grade. ZAP grading is a simple, uniform, objective, validated, cost-effective grading system and should be incorporated in endoscopic reporting of GERD patients.

KEYWORDS: Intestinal metaplasia; Z-line appearance; non-erosive esophagitis; Barrett's esophagus; Gastroesophageal reflux disease. 


\section{Introduction}

Endoscopic appearance of the Z-line at the gastroesophageal squamous-columnar junction has been classified as Z-line appearance (ZAP) grading system. ${ }^{1}$ The four graded ZAP grading system has been shown to correlate with the presence of intestinal metaplasia (IM) and the immunohistochemical cytokeratin 7/20 staining pattern at lower esophagus in both gastroesophageal reflux disease (GERD) and non-GERD population. ${ }^{2,3}$ Endoscopic assessment of ZAP grade has been traditionally used to determine the presence or absence of Barrett's esophagus (BE) at an early stage in patients of GERD by virtue of its strong correlation with prevalence of intestinal metaplasia. ${ }^{2}$ However, there is paucity of data in the literature regarding the clinical application of ZAP grading system in patients with GERD. Prevalence of GERD in the Indian population is about $7.6 \%$ and BE is rarely encountered. ${ }^{4}$ Hence we thought it to be worth while studying the prevalence of various ZAP grades in Indian GERD patients and comparing them with controls.

Among GERD patients, non-erosive reflux disease (NERD) accounts for approximately $70-90 \%$ of cases in the Asia pacific region including India. ${ }^{5}$ High resolution and high definition white light endoscopy, magnification endoscopy, chromoendoscopy, narrowband imaging and confocal laser endomicroscopy are some of the new techniques that are being tried currently across various institutions to detect microscopic esophagitis in patients with NERD. However, lack of standardization, complexity of interpretations, being costly and lack of widespread availability are some of the inherent limitations of these techniques. Z-line appearance grading is a simple, uniform, objective, validated, cost-effective grading system that can be done without the need for any specialized instruments. In previous studies ZAP grading has been proven to be clinically useful in patient with NERD. ${ }^{6,7}$ We did this study to find out the utility of ZAP grading in a resource limited setup like India where prevalence of NERD is high and BE is low.

\section{Materials and Methods}

\section{Study design:}

This was a hospital based prospective cross-sectional case-control study.

\section{Study population:}

\section{Inclusion criteria:}

We included 109 consecutive patients of GERD attending the outpatient department of a tertiary care center in Western India. Both males and females where included. Diagnosis was based on the symptom score calculated using a pre-validated GERD questionnaire. ${ }^{8,9}$ A symptom score was calculated based on the frequency and severity of heartburn and regurgitation in the past 1 week. In addition, data regarding age, sex, height, weight, smoking, tobacco chewing, alcohol drinking, drug intake, co-morbid illnesses, and previous abdominal surgery was also recorded.

\section{Symptom definitions used in our study to diagnose GERD included:}

1. Heartburn: defined as a burning sensation or discomfort behind the breastbone in the chest.

2. Acid regurgitation: defined as a bitter or sour tasting fluid coming in to the mouth.

The final score for each symptom, i.e., heartburn and regurgitation was obtained by multiplying the individual scores for severity and frequency. The total score was obtained by adding the final scores of individual symptoms. The final score ranged from 0 to 18 and GERD was diagnosed when the total score was $\geq 4$. Based on the final symptom score range of 4-8, 9-13, and 14-18, GERD was classified as mild, moderate, and severe respectively. The questionnaire used in our study is attached as a supplement file with this article.

In our study 102 controls were included who were patients in whom GERD was excluded based on GERD symptom score of $<4$. These were patients with vague 
abdominal symptoms, weight loss, anemia, etc. referred for routine upper gastro-intestinal endoscopy and had a normal study.

\section{Exclusion criteria for both cases and controls:}

Patients who were $\leq 18$ years of age, were on acid suppression therapy for 4 weeks before endoscopy, had history of upper gastrointestinal surgeries like gastrectomy, distal esophagectomy, gastro-jejunostomy, fundoplication, severe gastroparesis were excluded. In addition, pregnant patients, patients with contraindication to biopsy due to esophageal varices, bleeding disorders etc., patients with history of pill use and impaction, were also excluded.

\section{Sample size:}

Taking significance level of $\alpha=0.05$, equal sample size from two proportions $(\mathrm{r}=1)$, the probability $\mathrm{P} 1=0.47$ and $\mathrm{P} 2=0.65$ for ZAP I in non-GERD and GERD respectively were considered sufficiently different to warrant rejecting the hypothesis of no difference. Then the required sample size for two arms to achieve an $80 \%$ power $(\beta=0.2)$ was determined to be: cases $=104$, controls $=104$. The values of proportion of ZAP I in GERD and non-GERD used here were taken from the study by Wallner et al. ${ }^{3}$

\section{Endoscopy protocol followed in our study:}

Upper gastrointestinal (UGI) endoscopy was done using high definition white light endoscope (Olympus Video gastroscope GIF-H-170). During the examination, the landmarks including the diaphragmatic pinch, GEJ, and squamocolumnar junction were noted. Based on the presence or absence of erosive esophagitis cases were divided into non-erosive reflux disease (NERD) and erosive reflux disease (ERD). The Z-line was judged as distinct, blurred, or not detectable and evaluated based on the presence of any tongue-like protrusions or islands of columnar epithelium proximal to the Z-line. The length of any distinct tongue-like protrusions was noted. All the study subjects including cases and controls were assigned a ZAP grade.
ZAP grade used was ${ }^{2}$ :

1. Zap $0=$ sharp and circular (Figure 1A):

2. Zap $\mathrm{I}=$ an irregular Z-line with a suspicion of tongue-like protrusions and/or islands of columnar epithelium (Figure 1B);

3. ZAP II $=$ distinct tongues of columnar epithelium $<3$ $\mathrm{cm}$; the base of the tongue is shorter than the height (Figure 1C) and

4. ZAP III=distinct tongues of columnar epithelium $>3$ $\mathrm{cm}$, or a cephalad displacement of the Z-line $>3 \mathrm{~cm}$ (Figure 1D).

In addition, the patients of ERD were assigned Los Angeles (LA) grading of esophagitis 10 .

\section{Biopsy protocol followed in our study:}

Biopsy specimens for histologic evaluation were taken in cases as follows:

- one specimen from the cardia $2 \mathrm{~cm}$ below the gastroesophageal junction,

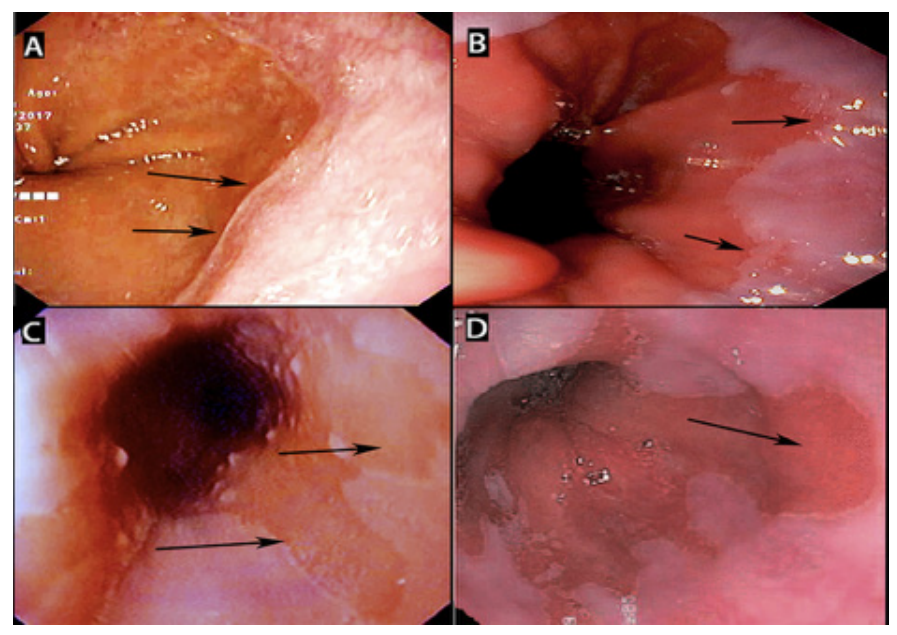

Figure 1(A): Shows ZAP grade '0' defined by sharp and circular Z-line without any tongues or island of columnar epithelium in lower esophagus. (B): shows ZAP grade ' $I$ ' defined by irregular Z-line with a suspicion of tongue-like protrusions (black arrow). ZAP grade ' $\mathrm{II}$ ' is defined by distinct tongues of columnar epithelium $<3 \mathrm{~cm}$ with the base of the tongue being shorter than the height (black arrow in figure C). ZAP grade ' $\mathrm{III}$ ' is defined as distinct tongues of columnar epithelium $>3 \mathrm{~cm}$ as shown in figure D (black arrow). 
- two specimens from the Z-line,

- two from the esophagus $2 \mathrm{~cm}$ above the Z-line,

- Two specimens from the corpus and

- Two specimens from antrum- within $2 \mathrm{~cm}$ of pylorus.

Specimens were taken from both the cardia and the Z-line to differentiate between IM of clearly gastric origin and IM of possible esophageal origin.

Two experienced pathologists independently examined the slides who were blinded to clinical and endoscopic data. Two sections stained with hematoxylin and eosin for histological assessment and one section stained with Geimsa stain for detection of Helicobacter pylori were examined. In addition, Periodic acid Schiff (PAS) stain and Alcian blue stain was used for detection of Intestinal metaplasia (IM). The esophageal biopsy was specifically assessed for evidence of microscopic esophagitis by noting for: necrosis/erosion, neutrophil/ eosinophil intraepithelial infiltration, basal cell hyperplasia, elongation of papillae, and dilation of intercellular spaces. In addition, presence of intestinal metaplasia and dysplasia was recorded in all the specimens.

The study was approved by institutional ethics committee.

Written informed consent was taken from each participant in the study.

\section{Statistical analysis:}

Categorical variables are presented as frequencies (percentages) and continuous variables as mean with standard deviation. Each categorical variable association with ZAP grade and type of GERD (NERD or ERD) was assessed by chi-square test. The Mann Whitney U test and Student $t$ test were used to compare non-parametric and parametric continuous variables, respectively. In addition, Spearman correlation was obtained between ZAP grading and LA grading. $\mathrm{P} \leq 0.05$ was taken as significant and all reported $\mathrm{P}$ values were two tailed. Likelihood ratio of ZAP grading in diagnosing NERD was also determined. All statistical analyses were performed using SPSS statistical package, version 22.0 statistical software (IBM, Armonk, NY).

\section{Results}

Table 1 depicts baseline demographic and clinical characteristics of GERD cases and controls. Mean age, sex distribution, presence of addiction (smoking, alcohol) and comorbidities (diabetes, hypertension) were similar in both the groups with no significant difference $(\mathrm{P}>0.05)$ except obesity which was more prevalent among cases as compared to controls $(\mathrm{P}=0.0014)$.

Figure 2 depicts bar diagram comparing prevalence of various ZAP grades among NERD cases, ERD cases and controls. The presence of ZAP grade 0 was significantly higher in controls than cases $(p=0.0001)$. NERD cases had significantly less severe ZAP grades (grades II and III) as compared to ERD cases( $\mathrm{p}=0.0001$ ).

Table 2 depicts the histological characteristics of ERD cases and NERD cases. Histologically presence of microscopic esophagitis at lower esophagus was similar in both ERD and NERD groups $(p=0.208)$. Although endoscopic Barrett's was seen in only 3 patients of GERD (ERD 2 and NERD 1), histologically intestinal metaplasia (IM) at lower esophagus was seen in 4 patients and at Z-line in 4 patients. At gastric cardia IM was seen in 8 patients which was more common in ERD than NERD cases but the difference was not significant $(p=0.068)$. At the gastric corpus, HP was significantly more common in NERD cases than ERD cases $(p=0.016)$. At the antrum,

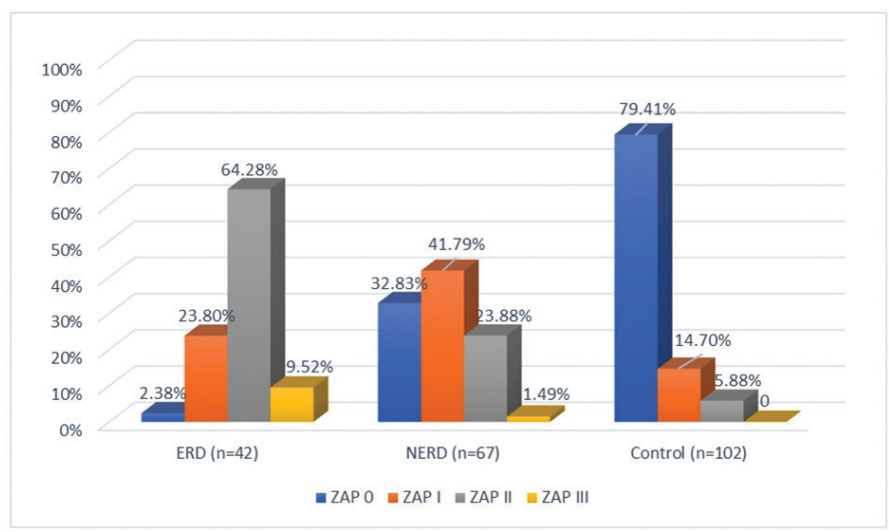

Figure 2: Bar diagram comparing the ZAP grade among controls, ERD cases and NERD cases clearly shows that controls had significantly more ZAP grade 0 and less ZAP grade I, II, III as compared to cases $(P=0.0001)$. Additionally, NERD cases had significantly less ZAP grade II, III and more ZAP 0 , I as compared to ERD cases $(p=0.0001)$. 
HP was more commonly seen in ERD cases as compared to NERD cases $(p=0.0001)$.

Comparison of histological features among various ZAP grades is shown in Table 3. Microscopic esophagitis was present in all the patients of ZAP III grade. It was more prevalent in ZAP II grade as compared to ZAP I grade ( $p=0.0023$ ). IM was significantly more common in ZAP III than ZAP II ( $\mathrm{p}=0.0004)$ and it was not seen in ZAP I and ZAP 0. It was on the basis of biopsies from Z-line showing tongue like projection in ZAP III. At

Table 1: Depicting the baseline demographic and clinical characteristics among ERD cases, NERD cases and controls.

\begin{tabular}{|c|c|c|c|c|}
\hline Characteristics & $\begin{array}{l}\text { ERD cases }(n=42) \\
\text { mean } \pm \text { SD or } n(\%)\end{array}$ & $\begin{array}{l}\text { NERD cases }(n=67) \\
\text { mean } \pm \text { SD or } n(\%)\end{array}$ & $\begin{array}{l}\text { Controls }(\mathrm{n}=102) \\
\text { mean } \pm \text { SD or } \mathrm{n}(\%)\end{array}$ & P values \\
\hline Age (years) & $39.9 \pm 12.7$ & $38.2 \pm 12.2$ & $40.6 \pm 13.9$ & 0.5 \\
\hline $\begin{array}{l}\text { Sex: } \\
\text { M } \\
\text { F } \\
\end{array}$ & $\begin{array}{l}24(57.14) \\
18(42.86) \\
\end{array}$ & $\begin{array}{l}30(44.77) \\
37(55.22) \\
\end{array}$ & $\begin{array}{l}54(52.94) \\
48(47.05 \%) \\
\end{array}$ & 0.4017 \\
\hline BMI $\left(\mathrm{kg} / \mathrm{m}^{2}\right)>25$ & $19(45.23 \%)$ & $23(34.32 \%)$ & $19(18.62 \%)$ & $0.0029 *$ \\
\hline Alcohol & $7(16.66 \%)$ & $10(14.9 \%)$ & $9(8.88 \%)$ & 0.3151 \\
\hline Diabetes & $12(28.57)$ & $13(19.40)$ & $16(15.68)$ & 0.2064 \\
\hline Smoking & $10(23.8 \%)$ & $15(22.38 \%)$ & $14(13.72)$ & 0.2229 \\
\hline Hypertension & $7(16.66)$ & $9(13.43)$ & $9(8.82)$ & 0.3700 \\
\hline $\begin{array}{l}\text { Duration of symptoms } \\
\text { (months) }\end{array}$ & $13.2 \pm 7.2$ & $14.3 \pm 8.5$ & NA & 0.46 \\
\hline GERD score & $9.166 \pm 3.286$ & $8.88 \pm 3.292$ & $1.82 \pm 0.943$ & 0.65 \\
\hline Mild GERD & $11(26.19)$ & $34(50.74 \%)$ & NA & $0.0196^{*}$ \\
\hline Moderate GERD & $18(42.85 \%)$ & $24(35.82 \%)$ & NA & 0.5944 \\
\hline Severe GERD & $13(30.95 \%)$ & $9(13.43 \%)$ & NA & 0.0485 \\
\hline
\end{tabular}

*denotes significant P values. GERD: Gastroesophageal reflux disease; ERD: Erosive reflux disease; NERD: Non-Erosive reflux disease; BMI: Body Mass Index; NA: Not applicable. Duration of symptoms and grading of GERD is only depicted among GERD cases in this table.

Table 2: Showing the histological characteristics among ERD cases and NERD cases.

\begin{tabular}{l|l|l|l} 
Histological Characteristic & $\begin{array}{l}\text { ERD cases }(\mathbf{n}=\mathbf{4 2}) \\
\mathbf{n}(\%)\end{array}$ & $\begin{array}{l}\text { NERD cases }(\mathbf{n}=\mathbf{6 7}) \\
\mathbf{n}(\%)\end{array}$ & $\mathbf{p}$ values \\
\hline $\begin{array}{l}\text { Lower Esophageal: } \\
\text { - Microscopic esophagitis } \\
\text { - Intestinal Metaplasia }\end{array}$ & $\begin{array}{l}32(76.19 \%) \\
3(7.14 \%)\end{array}$ & $\begin{array}{l}42(62.68 \%) \\
1(1.49 \%)\end{array}$ & 0.208 \\
\hline $\begin{array}{l}\text { Z-line: } \\
\text { - Intestinal Metaplasia }\end{array}$ & $3(7.14 \%)$ & 0 & 0.323 \\
\hline $\begin{array}{l}\text { Gastric Cardia: } \\
\text { - Intestinal Metaplasia }\end{array}$ & $6(14.28 \%)$ & $2(2.98 \%)$ & 0.105 \\
\hline $\begin{array}{l}\text { Gastric corpus: } \\
\text { - Helicobacter Pylori }\end{array}$ & $4(9.52 \%)$ & $21(31.34 \%)$ & 0.068 \\
\hline - Intestinal Metaplasia & $2(4.76 \%)$ & $6(8.95 \%)$ & $0.016^{*}$ \\
\hline $\begin{array}{l}\text { Antrum Biopsy: } \\
\text { - Helicobacter Pylori }\end{array}$ & $16(38.09 \%)$ & $4(5.97 \%)$ & 0.662 \\
\hline - Intestinal Metaplasia & $6(14.28 \%)$ & $2(2.98 \%)$ & $0.0001^{*}$ \\
\hline
\end{tabular}

*denotes significant P values. ERD: Erosive reflux disease; NERD: Non-Erosive reflux disease. 
the gastric corpus, HP was significantly more common in ZAP I as compared to ZAP II $(\mathrm{P}=0.0215)$ and it was not seen in ZAP III. On the other hand, HP at gastric antrum was seen similarly distributed in ZAP I, ZAP II and ZAP III $(p=0.3028)$.

Among symptomatic patients of GERD those who had NERD on endoscopy, usefulness of ZAP grading in making the diagnosis was determined. Compared to controls, NERD cases had significantly more positive ZAP grades (ZAP I, II, III) $(67.16 \%$ vs $20.58 \%, \mathrm{p}=0.0001)$. The likelihood ratio of ZAP grade to diagnose NERD among GERD cases was 3.26.
Table 4 illustrates the correlation between ZAP grading and LA grading in GERD cases. When severity of ZAP grade was correlated with the severity of LA grade, there was significant correlation (Spearman correlation factor, $r-0.478, \mathrm{p}<0.01$ ).

Table 5 demonstrates the correlation between GERD symptoms and ZAP grade. There was no significant correlation seen between severity of ZAP grade and severity of GERD as per the GERD symptom score $(\mathrm{p}=0.9004)$.

Table 3: Comparison of histological findings in GERD cases per the ZAP grading.

\begin{tabular}{l|l|l|l|l|l}
$\begin{array}{l}\text { Histological } \\
\text { Characteristic }\end{array}$ & ZAP 0 (n=23) & ZAP I $(\mathbf{n = 3 8 )}$ & ZAP II $(\mathbf{n = 4 3})$ & ZAP III $(\mathbf{n}=\mathbf{5})$ & P value \\
\hline Microscopic esophagitis & 0 & $27(71.05 \%)$ & $42(97.67 \%)$ & $5(100 \%)$ & For ZAP I vs II =0.0023* \\
\hline Intestinal metaplasia & 0 & 0 & $1(2.32 \%)$ & $3(60 \%)$ & For ZAP II vs III=0.0004* \\
\hline HP (body) & 0 & $17(44.73 \%)$ & $8(18.60 \%)$ & 0 & For ZAP I vs II=0.0215* \\
\hline HP (antrum) & 0 & $6(15.78 \%)$ & $13(30.23 \%)$ & $1(20 \%)$ & $\begin{array}{l}\text { For ZAP I vs ZAP II vs ZAP } \\
\text { III=0.3028 }\end{array}$ \\
\hline
\end{tabular}

GERD: Gastroesophageal reflux disease; ZAP grade: Z-line appearance. *denotes significant P values.

Table 4: Demonstrating the likelihood ratio of ZAP grade in diagnosing NERD among GERD cases.

\begin{tabular}{l|l|l|l} 
ZAP grade & NERD cases $(\mathrm{n}=\mathbf{6 7})$ & Controls $(\mathbf{n}=\mathbf{1 0 2})$ & Likelihood ratio \\
\cline { 1 - 3 } ZAPI-III (ZAP positive) & $45(67.16 \%)$ & $21(20.58 \%)$ & \multirow{2}{*}{ Likelihood Ratio=3.26 } \\
\hline ZAP 0 (ZAP negative) & $22(32.83 \%)$ & $81(79.41 \%)$ & \\
\hline
\end{tabular}

NERD: Non-Erosive reflux disease; ZAP-Z-line appearance.

Table 5: Showing correlation between ZAP grade and LA classification.

\begin{tabular}{l|l|l|l|l|l} 
& ZAP 0 & ZAP I & ZAP II & ZAP III & Total \\
\hline LA-Normal & $22(20.18 \%)$ & $28(25.68 \%)$ & $16(14.67 \%)$ & $1(0.91 \%)$ & $67(61.46 \%)$ \\
\hline LA-A & 0 & $8(7.33 \%)$ & $7(6.42 \%)$ & 0 & $15(13.76 \%)$ \\
\hline LA-B & $1(0.91 \%)$ & $2(1.83 \%)$ & $17(15.59 \%)$ & $1(0.91 \%)$ & $21(19.26 \%)$ \\
\hline LA-C & 0 & 0 & $3(2.75 \%)$ & $3(2.75 \%)$ & $6(5.5 \%)$ \\
\hline Total & 0 & 0 & 0 & 0 & 0 \\
\hline
\end{tabular}

LA- Los Angeles classification; ZAP- Z-line appearance classification

$L A-A$ : one or more mucosal breaks, no longer than $5 \mathrm{~mm}$, that do not extend between the tops of two mucosal folds; LA-B: one or more mucosal breaks, more than $5 \mathrm{~mm}$ long, that do not extend between the tops of two mucosal folds; LA-C : one or more mucosal breaks, that are continuous between the tops of two or more mucosal folds, but which involve less than 75\% of the circumference; $L A-D$ : one or more mucosal breaks, that involve at least $75 \%$ of the esophageal circumference. 


\section{Discussion}

Although ZAP grading system has been traditionally used in the West to determine the prevalence of BE by its correlation with IM, its use in Indian patients with GERD has not been described in the literature so far. It is a simple, objective and a validated grading system. ${ }^{2}$ In our study the severity of ZAP grade was significantly higher in GERD cases than controls. Among cases, it was significantly higher in ERD than NERD. However, the duration, frequency and severity of GERD symptoms as determined by the GERD symptom score did not correlate with ZAP grade. In the study by Kim et al, although ZAP grade was higher in ERD than NERD, they did not find any correlation between symptom severity and ZAP grade. ${ }^{6}$ Similar results were reported in other studies. ${ }^{7,11}$ This could be explained by presence of reflux hypersensitivity in some of the NERD patients with less severe ZAP grades. The likelihood ratio of diagnosing NERD in patients with positive GERD symptom score using ZAP grade was 3.26 when compared with controls suggesting a possible diagnostic role of ZAP grading in the Indian context. However, this needs further validation in large cohorts of patients from other parts of the country.

Indian patients with GERD have less severe symptoms and the prevalence of BE has been consistently low in various epidemiological studies 4,12,13. Extra esophageal manifestations of GERD is also uncommon in Indian and Asian subjects. ${ }^{4,14}$ In our study NERD cases were more prevalent than ERD (61.46\% vs $38.53 \%)$ which was consistent with previous reports from India. ${ }^{5}$

Although esophageal 24-Hr $\mathrm{pH}$-metry remains the gold standard for diagnosing NERD and differentiating it from reflux hypersensitivity and functional heartburn, lack of widespread availability, inadequate training and uniform reporting in India remains some of its limitations. Madan et al showed that combination of omeprazole challenge, endoscopy and histology can identify all the patients of GERD. ${ }^{9}$ They advocated that despite being the gold standard 24-hour pH-metry might not be a prerequisite for diagnosis of GERD in the Indian scenario. A validated GERD symptom based questionnaire as used in our study is a reasonable alternative to identify the burden of GERD as has been shown in previous studies from India. ${ }^{15}$ Since the ZAP grades in GERD patients were significantly higher than controls it could be a simple way to diagnose GERD in day to day practice.

LA classification and Modified Savary-Miller classification are some of the validated grading system in patients with ERD. ${ }^{10}$ Our study demonstrated a positive correlation between ZAP grading and LA classification in GERD patients with Spearman correlation coefficient of 0.478 . This suggests that ZAP grading system may be as useful as the LA classification in characterizing reflux esophagitis. Unlike ZAP grading LA classification has not been shown to correlate with IM and BE. Additionally, ZAP grading has high likelihood ratio of diagnosing NERD which cannot be done with LA grading. Thus, ZAP grading may be a better objective alternative to LA grading. This must be further validated in large multicentric studies.

Histologically microscopic esophagitis was seen significantly more in GERD patients with ZAP grade III and ZAP II as compared to ZAP I and ZAP 0. Thus, we propose that patients with positive GERD symptom score who have ZAP grade II or III on UGI endoscopy need not have lower esophageal biopsy as it may not provide additional useful information. Nandurkar et al, in their study did not find esophageal histology providing additional useful information over clinical assessment in patients with reflux symptoms undergoing upper gastrointestinal endoscopy16.However, this contrasted with other studies which reported that histological evaluation of the lower esophagus might provide an important diagnostic clue in endoscopically negative patients. ${ }^{7,17}$

Presence of IM at lower esophagus was also significantly more common in ZAP III as compared to ZAP II and it was not seen in ZAP 0 and ZAP I (Table 3). This was in corroboration with previous studies correlating ZAP grades with prevalence of IM. ${ }^{2}$ However, the number of patients with IM were small in our study to draw such conclusions. At the gastric corpus, HP was more common in NERD as compared to ERD. Similar findings were reported by Contractor et al, where they concluded that active corpus gastritis due to HP is inversely associated with erosive esophagitis among GERD patients18. Our study also demonstrated that 
HP at corpus was significantly more common in ZAP I as compared to ZAP II and III. This demonstrates that in addition to preventing macroscopic erosions at the lower esophagus HP associated corpus gastritis is also protective against severe morphological aberrations at Z-line in GERD patients.

There has been recent interest in the use of high definition endoscopy with I scan, magnification endoscopy, chromoendoscopy and narrow band imaging in NERD patients to identify patients with minimal change esophagitis. ${ }^{19}$ However, these are not yet standardized, easily available and are not yet validated. They are still at a premature stage for their use in GERD patients in day to day practice. It will be interesting to see any correlation between findings of these new techniques with ZAP grading among GERD patients.

Recently a multicentric prospective trial, recruited patients with irregular Z-line defined by endoscopic extent of esophageal columnar mucosa $<1 \mathrm{~cm}$ and intestinal metaplasia on biopsy. ${ }^{20}$ It showed that these cases do not develop high grade dysplasia or esophageal cancer within 5 years after index endoscopy. Thus, ZAP grading noted at index endoscopy may be a simple objective tool that may be added to the known armamentarium of conventional parameters used to stratify patients of GERD according to their risk of developing high grade dysplasia or esophageal carcinoma. Accordingly, we propose that ZAP grading may be incorporated in surveillance program in these patients in future.

Limitations of our study were lack of use of 24hour $\mathrm{pH}$ metry to further classify NERD patient groups, small number of patients with IM and lack of histological correlation with controls.

\section{Conclusion}

In Indian subjects with positive GERD symptom score ZAP grading is useful in diagnosing GERD, especially NERD. It correlates well with LA grading in GERD cases. At the lower esophagus, intestinal metaplasia was significantly more common with higher grades of ZAP. ZAP grading is a simple, uniform, objective, useful, validated, grading system and should be incorporated in endoscopic reporting of GERD patients.

\section{References}

1. Wallner B, Sylvan A, Janunger KG: Endoscopic assessment of the "Z-line" (squamocolumnar junction) appearance: reproducibility of the ZAP classification among endoscopists. GastrointestEndosc 2002; 55:65-69.

2. Wallner B, Sylvan A, Stenling R, Janunger KG. The esophageal Z-line appearance correlates to the prevalence of intestinal metaplasia. Scand J Gastroenterol 2000; 35: $17-22$.

3. Wallner B, Sylvan A, Stenling R, Janunger KG. The Z-line appearance and prevalence of intestinal metaplasia among patients without symptoms or endoscopic signs indicating gastroesophageal reflux. SurgEndosc 15:886-889, 2001.

4. Epidemiology and symptom profile of gastroesophageal reflux in the Indian population: report of the Indian Society of Gastroenterology Task Force: Bhatia SJ et al. Indian J Gastroenterology. 2011 May;30(3):118-27.

5. Goh KL. Gastroesophageal reflux disease in Asia: a historical perspective and present challenges. J Gastroenterol Hepatol 2011;26(Suppl 1):2-10.

6. Kim DH, Rhee PL, Cho DH, Chae JW, Kim JH, Kim JE, Son HJ, Kim JJ, Paik SW, Rhee JC: Diagnostic usefulness of the ZAP classification for non-erosive reflux disease (NERD). Korean J GastrointestMotil 2005; 11:97-103.

7. The Clinical Usefulness of the ZAP Classification for NonErosive Reflux Disease (NERD), Using Distal Esophageal Biopsy. BeomJin Kim, et al. Hepato-Gastroenterology 2009; 56:385-389.

8. Vigneri S, Termini R, Leandro G, et al. A comparison of five maintenance therapies for reflux esophagitis. N Eng J Med. 1995;333:1106-10.

9. Madan K, Ahuja V, Gupta SD, et al. Impact of 24-h esophageal $\mathrm{pH}$ monitoring on the diagnosis of gastroesophageal reflux disease: defining the gold standard. J GasteroenterolHepatol. 2005; 20:30-7.

10. Lundell LR, Dent J, Bennett JR, et al. Endoscopicassessment of oesophagitis: Clinical and functionalcorrelation and further validation of the Los Angeles classification. Gut 1999; 45:172-80

11. Labenz J, Jaspersen D, Kulig M, Leodolter A, Lind T, Meyer-Sabellek W, Stolte M, Vieth M, Willich S, Malfertheiner P: Risk factors for erosive esophagitis: a multivariate analysis based on the ProGERD study initiative. Am J Gastroenterol 2004; 99:1652-1656.

12. Lee HS, Jeon SW. Barrett esophagus in Asia: same disease with different pattern. ClinEndosc 2014; 47:15-22.

13. Chang CY, Cook MB, Lee YC, et al., Asian Barrett's 
Consortium. Current status of Barrett's esophagus research in Asia. J Gastroenterol Hepatol 2011; 26:240-6.

14. Jung HK. Epidemiology of gastroesophageal reflux disease in Asia: a systematic review. J NeurogastroenterolMotil 2011; 17:14-27.

15. P. K. Sharma, V. Ahuja, K. Madan, S. Gupta, A. Raizada, M. P. Sharma. Prevalence, severity, and risk factors of symptomatic gastroesophageal reflux disease among employees of a large hospital in Northern India. Indian J Gastroenterol (May-June 2011) 30(3):128-134.

16. Nandurkar S, Talley NJ, Martin CJ, Ng T, Adams S. Esophageal histology does not provide additional useful information over clinical assessment in identifying reflux patients presenting for esophagogastroduodenoscopy. Dig Dis Sci 2000;45:217-224.

17. Funch-Jensen P, Kock K, Christensen LA, Fallingborg J, Kjaergaard JJ, Andersen SP, Teglbjaerg PS: Microscopic appearance of the esophageal mucosa in a consecutive series of patients submitted to upper endoscopy. Correlation with gastroesophageal reflux symptoms and macroscopic findings. Scand J Gastroenterol 1986; 21:65-69.

18. Contractor QQ, ulHaque I, Saka H, Contractor TQ. Corpus gastritis and erosive oesophgagitis: a report from the Middle East. Indian J Gastroenterol. 2006;25:295-7.

19. Pungrasamee A, Pisespongsa P, Praisontarangkul O, Thongsawat S, Leerapun A, Chitapanarux T. Reliable Endoscopic Findings of Minimal Change Esophagitis for Diagnosis of Non-erosive Reflux Disease (NERD) Using High Definition Endoscopy with I-scan. Thai J Gastroenterol 2012; 13(2): 87-92.

20. Thota PN, Vennalaganti P, Vennelaganti S, Young P, Gaddam S, Gupta N et al.Low Risk of High-Grade Dysplasia or Esophageal Adenocarcinoma Among Patients With Barrett's Esophagus Less Than $1 \mathrm{~cm}$ (Irregular Z Line) Within 5 Years of Index Endoscopy.Gastroenterology. 2017 Apr;152(5):987-992. 